\title{
A Latent Growth Model Evaluation of the Comprehensive, Integrated HIV Prevention Program Effect on Excessive Alcohol Risk Awareness and Excessive Alcohol Consumption Risk
}

\author{
Meya YB Mongkuo ${ }^{1 *}$, Maurice Y Mongkuo ${ }^{2}$ and Nicole Lucas ${ }^{3}$ \\ ${ }^{1}$ Virginia State University, Department of Psychology, USA \\ ${ }^{2,3}$ Fayetteville State University, USA
}

*Corresponding author: Meya YB Mongkuo, Department of Psychology, Virginia State University, USA.

Received Date: October 01, 2020

Published Date: October 28, 2020

\begin{abstract}
Aim: Since the early 1980s, excessive consumption among minority young adults (age 18-24 years old) became a severe public health concern in preventing the prevalence of HIV infection. In the United States, minority young adult population have a higher excessive alcohol consumption rate of HIV infection disproportionately infected with the disease than any other racial group. The continued spread of HIV among this vulnerable population has led to a need to identify risk and protective factors and evidence-based prevention strategies to reduce the transmission of the disease. A popular approach is the comprehensive, integrated HIV prevention program (CIHPP), a program based on the ecological epistemology framework. This framework views risk factors as a multilevel concentric system, including the individual, family, community, and societal levels. The theory asserts that any meaningful prevention strategy should examine the effect of these different levels on the population of interest. This study aims at testing the effectiveness of the CIHPP in raising awareness and excessive alcohol consumption risk of minority young adults.
\end{abstract}

Methods: Data on excessive alcohol risk awareness and alcohol consumption was collected from a survey of a random sample of minority young adults who participated in 24 months of CIHPP. Change in their excessive alcohol risk awareness and use was recorded during the 24 months of their participation in CIHPP. The impact of the CIHPP on excessive alcohol risk awareness and alcohol consumption were evaluated using the latent growth curve model within the framework of the structural equation modeling procedure.

Results: The findings reveal that the CHIPP was effective in raising the excessive alcohol consumption risk awareness and reducing alcohol consumption of the participants. There was a significant inter-individual difference in the original score of excessive alcohol risk awareness between the participants and progressed through 24 months of CIHPP intervention. Regression analysis revealed that excessive alcohol consumption risk awareness of female participants was higher than their male counterparts. There were significant inter-individual differences or heterogeneity in alcohol consumption in the original score of excessive alcohol consumption between the participants. Regression analysis revealed that the male participants consumed more alcohol than their female counterparts.

Conclusion and Discussion: The CIHPP was effective in increasing the awareness of excessive alcohol consumption risk and reducing excessive alcohol consumption among minority young adults. The alcohol awareness of minority young female adults was higher than their male counterparts during the 24 months implementation of the CIHPP. These findings confirmed our research hypotheses and consistent with the results of previous research on evidence-based interventions.

Keywords: Alcohol consumption risk awareness; Excessive alcohol consumption risk; Latent growth curve modeling; Structural equation modeling; Comprehensive HIV prevention program 


\section{Introduction}

It has been almost four decades when HIV infection became a severe public health problem. Since then, infectious disease experts worldwide have been working without finding a vaccine to immune people from the disease. So the focus has shifted from developing a vaccine to identifying the most effective evidence-based prevention strategies to slow the spread of the disease. In 2017 an estimated 5.1 million or $14.8 \%$ of young adults aged 18 to 25 were diagnosed with substance use disorder [1,2]. Other researchers have focused on identifying evidence-based risk and protective factors and prevention strategies to slow the disease [3-8]. Research has found excessive alcohol consumption destabilize the normal functioning of virtually all vital organs that regulate behavior, including the nervous system, the immune system, the digestive system, the endocrine system, and the circulatory system $[3,9]$. These hormones control metabolism and energy levels, electrolyte balance, growth and development, reproduction, responses to and appropriate coping with changes in the internal and external environments, such as changes in temperature and the electrolyte composition of body fluids, and response to stress, anxiety, and injury [3,5-8,10].

Both acute and chronic alcohol consumption induces hormonal disturbance of the endocrine system that disrupts the body's ability to maintain homeostasis leading to various disorders, including cardiovascular diseases, reproductive deficits, immune dysfunction, certain cancers, bone disease, and psychological and behavioral disorders. Research has also found that indulgence in excessive alcohol consumption does not only harm the hypothalamicpituitary-adrenal (HPA) axis, but practically all the hormonal systems of the body, including the hypothalamic-pituitary-gonadal (HPG) axis Sakar DK, Gibbs DM [5], the hypothalamic-pituitarythyroid (HPT) axis [11], hypothalamic-pituitary-growth hormone/ insulin-like growth factor-1 (GH/IGF-1) axis [13-15], and the hypothalamic-posterior pituitary (HPP) axis [3,4,17-19].

A recent review of studies on youth and adolescents also suggests that hypothalamic-pituitary-adrenal (HPA) axis dysfunction and exposure to stress are critical components that interact to convey risk for developing attention deficit disorder (AUD) [20]. For example, several randomized clinical trials (RCT) have found that excessive alcohol consumption harms the normal functioning of the nervous and endocrine systems, both of which are responsible for proper communication between various organs and cells of the body to maintain a stable internal environment or homeostasis [2122]. Interference of the normal functions of these communication systems sets in motion a series of adverse physiological activities, including disruption of the hormonal control of metabolism and energy levels, electrolyte balance, growth and development, and reproduction of the body. These disruptions, in turn, inhibits the body's ability to respond to effectively and appropriately adapt to changes in body temperature or the electrolyte composition of the body's fluids, response to stress and injury, and psychological and behavioral disorders [1, 22-24]. As for the nervous system, excessive consumption of alcohol disrupts the vital hormonal flow of the hypothalamic-pituitary-adrenal HPP axis that is responsible for cognitive brain functioning. The HPP axis includes two neuropeptides called arginine vasopressin (AVP) and oxytocin.

Other research has focused on the progressive alterations in the HPA axis function crucial for understanding the underlying brain mechanisms of substance use, including excessive alcohol consumption disorders. These studies found that in contrast to mood and affective disorders, alcohol dependence has a biphasic effect on HPA axis dynamics as a person traverses through the various phases of heavy hazardous drinking, including dependent alcohol, withdrawal, abstinence, and relapse. Generally speaking, these developmental stages seem to be mirrored by a shift between hyper- and hypo-responsiveness of the HPA axis to stressful events [25]. For example, hyper-responsiveness has been identified in people with a family history of alcoholism $[8,10]$, a population that is at increased risk for alcohol dependence (Windle 1997). Thisfinding raises the question of whether heightened stress responsivity is clinically meaningful to the development of alcoholism. This view is supported by studies showing that cortisol responsivity correlates with the activity of the regulatory function of the nervous system called the mesolimbic dopaminergic pathway, which is a central neural reward pathway $[8,26]$. The transition to alcohol dependence leads to compensatory allostatic mechanisms result in injury to HPA axis function and elevation of stress peptide levels (e.g., corticotropin-releasing factor (CRF) in brain regions outside the hypothalamus. Allostasis refers to the process through which various biological processes attempt to restore homeostasis when an organism is threatened by multiple types of stress in the internal or external environment $[3,27]$. Allostatic responses can involve alterations in the HPA axis function, the nervous system, various signaling molecules in the body, or other systems. Allostatic changes in HPA axis function have been posited to, among other things, injure brain reward pathways, contribute to depressed mood (i.e., dysphoria) and craving, and further contribute to the maintenance of problem drinking behavior.

A close examination of the physiology of the hypothalamicpituitary-adrenal (HPA) axis reveals that the body responds to stress with automatic, allostatic processes aimed at returning critical systems to a set point within a narrow range of operation that ensures survival $[3,4]$. These automatic processes consist of multiple behavioral and physiological components. Perhaps the best-studied element in the stress response in humans and mammals is the activation of the HPA axis. This line of inquiry has found that the neurons in the paraventricular nucleus (PVN) of the hypothalamus release two neurohormones-CRF and arginine vasopressin (AVP)-into the blood vessels connecting the hypothalamus and the pituitary gland (i.e., hypophysial portal blood). Both hormones stimulate the anterior pituitary gland to produce and secrete adrenocorticotropic hormone (ACTH) into 
the general circulation. The ACTH, in turn, induces glucocorticoid synthesis and release from the adrenal glands located atop the kidneys.

The main glucocorticoid in humans is cortisol, which frequently is used as model systems to investigate the relationship between stress and alcohol use, which is corticosterone. Hypothalamic activation of the HPA axis modulated a variety of brain signaling (i.e., neurotransmitter) systems. Some of these systems have inhibitory effects (e.g., g-aminobutyric acid [GABA] and opioids), whereas others have excitatory effects (e.g., norepinephrine and serotonin) on the PVN. These effects suggest that the central nervous system (CNS) and the hormone (i.e., endocrine) system are tightly interconnected to coordinate glucocorticoid activity [28]. The HPA system carefully modulates through negative-feedback loops designed to maintain predetermined hormone levels (i.e., setpoints) and homeostasis. To this end, Hermann [28] asserts that secretion of CRF, AVP, and ACTH in part controlled by sensitive negative feedback exerted by cortisol at the level of the anterior pituitary gland, PVN, and hippocampus.

Iovino [18] suggest that there are two types of receptors for cortisol-mineralocorticoid (type-I) and glucocorticoid (typeII) receptors-both of which participate in the negative feedback mechanisms. Cortisol binds more strongly (i.e., has higher binding affinity) for the mineralocorticoid receptors (MRS)1 than the glucocorticoid receptors (GRs). Because of this difference in a critical relationship, the MRS help maintain the relatively low cortisol levels circulating in the blood during the regular daily (i.e., circadian) rhythm. Only when the cortisol concentration is high (e.g., during a stressful situation) does it bind to the GRs with lower affinity; the resulting activation of the GRs terminates the stress response. This delicate negative feedback control mechanism maintains the secretion of ACTH and cortisol within a relatively narrow bandwidth [29]. This process is a critical homeostatic mechanism because it regulates too much or too little exposure AVP secreted in response to osmotic stimuli. Also, it restricts the concentration of dissolved molecules (i.e., osmolality) in the blood fluid by retaining water in the body and constricting blood vessels $[18,29]$. Some AVP is released directly in the brain, and research suggests that it play an essential role in social behavior, sexual motivation and pair bonding, as well as maternal response to stress (Dorin et al. 2003; Ehrenreich et al. 2010).

Excessive alcohol consumption lowers the level of AVP to the brain leading to impaired cognitive performance (Laczi 1987). Like AVP, oxytocin is produced by both magnocellular and parvocellular neurons of the hypothalamus and functions both as a peripheral hormone and a signaling molecule in the central nervous system Buijs [30] to regulate adaption of the body to respond effectively to internal physiological and environmental changes or disruptions. Research on people with a history of excessive alcohol consumption shows that hyperresponsiveness of the stress response is mediated by the HPPA axis [8], leading to mental health problems. Research on animals found acute ethanol administration to rats increased plasma ACTH and corticosterone levels by enhancing CRF release from the hypothalamus [31-32]. Chronic alcohol consumption is associated with anxiety-producing (i.e., anxiogenic) Behavior [33]. Collectively, these studies show that excessive or chronic alcohol consumption attenuates basal ACTH and corticosterone levels and increases stressful and anxiogenic behaviors. Other studies have found an association between excessive alcohol consumption and depression among young adults [34-36].

\section{Rate of excessive alcohol consumption in Cumberland, North Carolina}

The Center for Disease Control and Prevention (CDC) reports that Cumberland County's rate of people drinking five or more drinks of alcohol in one seating of $11.2 \%$ is higher than North Carolina State's rate of $10.5 \%$. Disease prevalence data and study findings suggest that our target populations have a more severe than expected alcohol abuse problem that makes them vulnerable to alcohol-related HIV infection. The CDC also estimates that Cumberland County's rate of people drinking five or more drinks of alcohol in one seating of $11.2 \%$ is higher than North Carolina State's rate of $10.5 \%$ [2]. The North Carolina Department of Health and Human Service (NCHHS) reports that Cumberland County continues to battle against sexually transmitted diseases. North Carolina Department of Health and Human Services reports that in 2013, there 1,339 persons living with HIV infection (PLWHI) in Cumberland County. Of this total, 866 had HIV, and 473 had AIDS. There were 158 PLWHI young adults ages 15-24 years old, representing $0.6 \%$ with a corresponding HIV infection rate of 27.7 per 100,000 population in Region 5, which includes Cumberland County. This HIV infection rate is higher than North Carolina's rate of 25.7 per 100,000 people. Desegregating the PLWHI.rate by regionshows that the rate in Region 5, which includes Cumberland County by race/ethnicity,reveals that except for Hispanics and Asian/Pacific Islanders, the rate of PLWHI for Region 5 was higher than that of North Carolina, with the PHLI.Rate and percent of Americans of $4.9 \%$ and 189.6 per 100,000 population areseven times higher than North Carolina's $0.7 \%$ and 175.2 per 100,000 people; African Americans were $69.4 \%$ and 710.4 per 100,000 people compared to North Carolina's $65.4 \%$ and 857.8 per 100,000 people. This prevalence data suggest that higher than expected level of HIV infection among our target populations, and hence a need for evidence-based intervention.

\section{Rate of HIV infection in cumberland county, north carolina}

North Carolina Department of Health and Human Services report that in 2013, Cumberland County had 97 newly diagnosed HIV infections, which rank 3rd among all North Carolina Counties in newly diagnosed HIV infection rate with $26.0 \%$ HIV infections per 100,000 population (97 cases) compared to NC. rate of $15 \%$ 
per 100,000 people. From 1983 to 2013, Cumberland County had a cumulative number of HIV cases of 2,087, which ranks 6th out 100 Counties in North Carolina. During the same period, the County had 910 increasing cases of AIDS, which ranks 6th among the 100 counties in North Carolina. North Carolina State Center for Health Statistics (NCSCHS) reported that during the period 20072011, Cumberland County's HIV rate of infection of 27.3/100,000 population was 1.54 times higher than the State of North Carolina's HIV infection rate of 17.7 per 100,000 people. Also, NCSCHS reported that during 2007-2011, Cumberland County's total AIDS rate of 3.4p/100,000 population was 1.7 times higher than North Carolina State's overall AIDS rate of $2.0 \mathrm{p} / 100,000$ population and $13 \%$ higher than all its peer counties, except for one (Mecklenburg County) in the State of North Carolina.

\section{Theoretical Framework}

Theoretical approaches to prevention have three primary assumptions. First, they view prevention as a proactive process by which conditions that promote the well-being of an individual. Prevention activities empower individuals and communities to meet the challenges of life events and transitions by creating conditions and reinforcing individual and collective behaviors that lead to healthy communities and lifestyles. Second, prevention requires multiple processes on multiple levels to protect, enhance, and restore the health and well-being of high sexual risk populations. Such as minority young adults in Cumberland County. Third, prevention involves an understanding of risk and protective factors that vary among individuals, age groups, racial and ethnic groups, communities, and geographic areas.

Theories, models, and data that allow for the explanation and understanding of sexual risk and protective factors at several levels of social aggregation-community, school, peers, family, and the individual's characteristics-provide a rational approach to designing appropriate prevention strategies and programs. Risk factors exist in clusters rather than in isolation. Research has shown that these multiple risk factors have a synergistic effect (i.e., the interactions between these risk factors have a more significant impact than any single risk factor) alone. For example, some of the behaviors that put people at heightened risk of contracting and spreading HIV are excessive alcohol consumption, illicit substance, and tobacco use, and having sex with multiple sex partners.

\section{The Comprehensive HIVPrevention Program (CIHPP)}

CIHPP is essentially a derivative of Bronfenbrenner's [37] ecological epistemology framework, which asserts that health risk behaviors such as excessive alcohol consumption, involves complex interactions between social and biological factors [38] March \&Susser, 2006; Dalhberg\& Krug, 2002; [39] Schiberner et al., 2001. This approach to health risk behavior prevention is considered the most effective evidence-informed strategy to prevent the spread of HIV and other infectious diseases among at-risk populations. This framework emanates from Jessor's [40] problem behavior theory
(PBT), which proposes interrelated concentric domains of risk factors beginning with the individual level, the neighborhood or community level, and societal level $[37,39]$. Specifically, the states that young adult health risk factors consist of a personality system, social environment, and behavior. The approach extends to the domain of psychosocial theory that views health risk behaviors as co-occurring [40-41] among young adults. Hence, assessing the effectiveness of prevention programs should include examining the association between externalizing problems (such as alcohol consumption) and internalizing problems (such as depression, anxiety, cognitive impairment, and disruptive behavior). Therefore, as suggested by [37], effective prevention strategies should identify and address the prevention of sexually transmitted infections among high-risk individuals and communities at all four levels (i.e., individual, interpersonal, organization, and societal).

The individual level is considered the microsystem where individuals work within their family and home environment, school and peers, work-peer networks, peer support, family support, parental mentoring, and parental involvement in health risk behaviors networks. [37,40-42]. This individual-level characteristic is nested within the broader community, consisting of community norms, attitudes regarding health risk behavior, cultural standards, gender norms, spiritual and religious norms, and ideological and political norms. The prevalence of individual-level health risk behaviors may include having multiple sex partners, having sex without condoms, having concurrence partnerships, sharing infected needles, and readily available alcohol, illicit substance, and tobacco. The prevalence of interpersonal risk behavior is social and sexual network structure (i.e., network size, density, mixing, and turnover) and compositional factors (i.e., characteristics of network members) that influence vulnerable to HIV infection and transmission such as minority young adults HIV transmission [43].

Community-level risk factors include the density of alcohol, tobacco, illicit substance and tobacco outlets, and community social and economic disadvantages, crime, and homelessness [4447]. Societal level sexual risk factors consist of public policies that shape the environment of the community, such as policies that promote high density of alcohol and other risky sexual behavior products outlets in poor and minority neighborhoods, leading to segmentation of drinkers in hot spots for HIV risk behaviors and HIV transmission [46]. Also, societal health risks may include institutional racism, stigma, segregation, formal and informal public policies, and religious and cultural norm $[44,47]$.

The macro-policy level may also include the biological and physiological status of essential systems of the body that regulate behavior, including the nervous system, endocrine system, the digestive system, immune system, and renal system. The macropolicy level consists of advertisements and marketing policies related to health risk behaviors [37,39]. Hence, effective prevention strategies and procedures should include considering all these 
multiple interrelated spheres of influence on behavior to achieve desired health outcomes.

The ecological epidemiology framework of the comprehensive HIV prevention program germane to our study implies identifying the prevalence of HIV infection and transmission rates in the target population by conducting needs assessments of measurable constructs at each level or domain of influence, at cross-level connections at both the micro and macro levels, as well as by examining the macrosocial and microsocial or protective factors (risk regulators) that can either constrain or promote the occurrence of individual-level behavior associated with the risk of HIV infection [48]. The needs assessments, in turn, provide objective data for developing a strategic HIV prevention plan for the target population and community. So far, no research that we know of have has validated the psychometric properties of the expected outcome of CHIPP, as well as an evaluation of the desired results of this prevention intervention strategy, including an increase in excessive alcohol consumption risk awareness, decrease in excessive alcohol consumption. So far, there has been no study that we know of has determined the change in behavior in the behavior of participants over time using the latent growth curve model within the framework of structural equation modeling.

\section{Purpose of the Study}

The purpose of this study is to begin a line of inquiry to fill this gap in research by examining the effectiveness of the Comprehensive Integrated HIV Prevention Program in raising awareness and decreased the involvement of risky sexual behaviors among minority young adults. We expect that the study to provide public policymakers, stakeholders, and practitioners with reliable and valid policy-relevant information relied upon in designing efficient and effective public policies to reduce the spread of HIV infection among this vulnerable population.

\section{Research question}

This study sought to provide an empirically-ground answer to the following two research questions:

- What is the effectiveness of the comprehensive, integrated HIV prevention program in raising excessive alcohol consumption risk awareness of minority young adults?

- What is the excessiveness of the comprehensive, integrated HIV prevention program in reducing excessive alcohol consumption of minority young adults?

- $\quad$ Research Hypothesis

- The Comprehensive Integrated HIV Prevention Program (CIHPP) effectively increases excessive alcohol consumption risk awareness of minority young adults.

- The (CIHPP) is effective in reduces excessive alcohol consumption among minority young adults.

\section{Materials and Method}

\section{Research design}

The study used a pre-experimental One-shot latent growth curve (LGC) model case Study Design) [49-53]. Figure 1 displays a schematic representation of the design. Treatment Post-test $X$ OT1 OT2.... OTn.

Where $\mathrm{X}$ is the participation level of minority young adults in the comprehensive, integrated HIV prevention program, 02 is the level of minority young adults'excessive alcohol consumption risk awareness and excessive alcohol consumption. A limitation of this type of research design is the lack of a control group. However, latent growth curve modeling within the framework of the structural equation model modulates this limitation by having six main advantages over the traditional longitudinal research design. Specifically, the LGC modeling approach to evaluating change has six important unique features that make it superior to other longitudinal procedures in assessing domain outcomes change over time. First, the method can accommodate anywhere from three to thirty waves of longitudinal data equally well. Indeed, Willett $(1988,1989)$ has shown that the more waves of data collected, the more precise the estimated growth trajectory and higher will be the reliability of the measurement of change. Second, there is no requirement of the time between each wave of assessments to be equivalent, which suggests that the LGC modeling approach can comfortably accommodate irregularly spaced measurements with the caveat that the same set of occasions measures participants.

Third, personal change can be represented by either a linear growth or a non-linear growth trajectory, although linearity is usually assumed. The assumption is tested, and the model respecified to address curvilinearity if need be. Fourth, in contrast to traditional longitudinal methods used in measuring change, the LGC allows not only for estimating measurement error and accounts for autocorrelation but also for fluctuation across the time when the test for the assumptions of independence and homoscedasticity is untenable. Fifth, the multiple predictors of change can be included in LGC as fixed or time-varying [54]. Finally, the independence of measurement error variances and homoscedasticity of measurement can be tested by comparing nested models [54].

\section{Participants and Procedure}

Participants in this study were a random sample of minority young adults (18-24 years old) living in a high prevalent community in the southeastern United States who volunteered to participate in the study. Upon getting the Institutional Review Board's (IRB) approval of the study questionnaire and proposal, culturally and linguistically appropriate announcements and advertisements were was send out to residents of the high HIV prevalence community through various minority young adult outlets, including social media, radio, print media, community organizations, word-ofmouth, and distribution of flyers inviting them to attend community 
health events and participate is a healthy living event.

Minority young adults who volunteered to participate in the study were informed that a survey would be conducted periodically over 24 months to obtain their opinion about key risky behaviors, such as excessive alcohol consumption risk awareness, excessive alcohol consumption, that may predispose people to HIV infection. The minority young adults who agreed to participate were given a linguistically and culturally appropriate consent form to read, sign, and return. The consent stated that their participation in the survey was strictly voluntary; they may either opt not to participate in the study or not provide a response to any of the statements; their identity will not appear in any report; a $\$ 30$ gift card will be given to them as an incentive for fully participating in the surveys. The minority young adults who agreed to participate in the study were provided with a linguistically appropriate consent form to read, sign, and date. The consent form explained to the community residents that their participation was voluntary and that their identity would be kept strictly confidential, and their names would not appear in any report.

The survey instrument used in this study is the National Minority Substance/HIV Prevention Initiative Adult Questionnaire approved on March 15, 2016, by the United States Office of Management and Budget, containing a total of health risk-related constructs and over 56 items. The Questionnaire included over 100 constructs, 70 measurement items, and demographic information of the participants. Upon the Institutional Review Board (IRB) approval, we administered the survey to the participants who volunteered, read, and signed the consent form. We adhered to all American Psychological Association research guidelines. The survey was anonymous in that no identifying information was connected to individual participants or included in the study data set. The participants completed the study in less than 25 minutes during the HIV prevention events and returned them before leaving. A total of 518 minority young adults participated in the survey, and 498 of them completed the entire survey representing a 96 percent response rate. A small sample of the to check for internal consistency reliability of the items measuring the three constructs of interest using Cronbach's Alpha Reliability test. The test produced five items measuring excessive alcohol risk awareness, and two items measuring excessive alcohol consumption.

\section{Measures}

The items measuring excessive alcohol consumption risk awareness and alcohol consumption were: Alcohol consumption risk awareness was measured by three items such as "How much do people harm themselves physically or in other ways when they have five or more drinks of an alcoholic beverage once or twice a week? The items were scored on an ordinal Likert Scale ranging from 0 days $=0$ to 30 days $=30$. Excessive alcohol consumption was measured by five items, such as, "Have you ever felt bad or guilty about your drinking? The questions were scored on an ordinal
Likert Scale ranging from 0 days $=0$ to 30 days $=30$.

\section{Statistical Analysis}

This study used the latent growth curve modeling (LGC) within the SEM framework to evaluate the intraindividual and interindividual change of excessive alcohol consumption risk awareness and excessive alcohol consumption CIHPP participant overtime. The hierarchical levels to use in assessing invariance consist of

- Configural Invariance test to determine if the same factor structure exists in all groups.

- Metric Invariance to test whether the loading estimates are equal in all group, which allows comparisons of relationships.

- Scalar Invariance to verify whether the intercept terms for all equations are similar in all groups which allow for comparisons of means.

- Factor Covariance Invariance to test whether the covariances matrix among latent constructs is the same in all groups.

- Factor Variance Invariance to test whether the factor variances are the same in all groups.

- $\quad$ Error Variance Invariance to test whether error variance terms are the same in all groups.

The analytic method used to assess the psychometric properties of the National Minority SA/HIV Prevention Initiative Adult Questionnaire (NMSPIAQ) consists of four interrelated SEM procedures. First, Exploratory Factor Analysis (EFA) to assess the factorability of each factor and evaluate the internal consistency (i.e., Cronbach's alpha) of the psychometric properties of NMSPIAQ using SPSS version 26.0. Second, single group Confirmatory Factor Analysis (CFA) of NMSPIAQ determines the construct and content validity of NMSPIAQ. Third, a series of Multi-group C.F.A. to test the invariance of NMSPIAQ across static factors groups. Fourth, Latent Growth Curve (LGC) modeling within the SEM framework using Analysis of Moment Structure (AMOS) version 26.0 to answer questions about systematic intra-individual BOP innate and inter-individual BOP innates differences in change over time of a minority young adult's likelihood of excessive alcohol consumption. AMOS statistical software version 26.0 was used to conduct the second through the fourth analysis. A description of each of these procedures is presented below.

Exploratory factor analysis - The first phase of the data analysis involved assessing the reliability or internal consistency of the primary CIHPP outcome constructs by performing an exploratory factor analysis (EFA) to determine the meaningful factor loading structure of the items or observed variables were measuring the CIHPP outcome constructs. The EFA began by checking the assumptions necessary for proceeding with factor analysis. The 
check involved assessing the degree of intercorrelation of the items from both the overall and individual variables perspectives. The overall measure of intercorrelation was evaluated by

- Computing the partial correlation or anti-image correlation among the variables, with small values indicating the existence of "true" factors in the data [55].

- Performing Bartlett's Test of Sphericity, with significant approximate chi-square $(\chi)$ indicative of significant correlation among at least some of the construct's observed variables;

- $\quad$ Estimating the Kaiser-Meyer-Olkin Measure of Sampling Adequacy (MSA) value, with MSA values above .50 considered acceptable to proceed with factor analysis [55].

The variable-specific measure of intercorrelation was assessed by estimating the Kaiser-Meyer-Olkin (KMO) measure of sampling adequacy (MSA) value for each observed variable or item with values below .50 considered to be unacceptable [55-56]. The variable with the lowest MSA value was deleted, and the factor analysis was repeated. This process continued until all the observed variables had acceptable MSA values, and a decision was made to proceed with factor analysis. Principal component factor analysis applying the varimax rotation was used to reduce or organize the item pool into a smaller number of interpretable factors. The number of factors was determined by joint consideration of Cattell's (1966) scree plot, a priori, and the percentage of factors to be extracted criteria [55]. The latent root residual (eigenvalue) criterion was considered inappropriate if the number of observed variables fells below or outside the acceptable range of 20 to 50 [55]. [57] principle of simple structure using pattern coefficients of absolute 0.35 as the lower bound of influential per factor and interpretability of the solution used to determine the final solution Lambert \& Durant, 1974; [55]. After rotation, variables with crossloading and communalities lower than .50 were deleted [55].

The second step of the analysis involved reviewing the items measuring each dynamic factor by calculating the internal consistency estimates (Cronbach's alpha) for the items representing each factor retained from the exploratory factor analysis procedure. Cronbach's alpha of 0.6 was considered the minimum acceptable level of internal consistency for using a factor [55]. For factors with Cronbach's alpha below this minimum benchmark, the internal consistency of the factor was improved by identifying and removing items with low item-test correlation and item-rest correlation. The factor was deleted if no improvement in the reliability score occurred.

\section{Single group confirmatory factor analysis}

After establishing the reliability of the CIHPP expected outcomes constructs, the constructs were validated by performing a single group CFA. This validation involved testing for the factorial stability of each CIHPP outcome construct. This test aimed to determine the extent to which items designed to measure each CIHPP outcome factor (i.e., latent construct) do so. Because the analysis was performed on original data and not data summary, missing data were accommodated using the full information maximum likelihood (FIML) procedure. This procedure allowed the maximum likelihood estimation to be performed on a dataset containing missing data, without any form of imputation [58]. Several indices were used to evaluate the goodness of fit of the 6-factor orthogonal CIHPP measurement model.

The guidelines for determining model fit consisted of adjusting each index cutoff values based on model characteristics as suggested by simulation research that considers different sample size, model complexity, and degree of error in the model specification as a basis for determining how various accurate indices performed [55,59]. The model's absolute fit was assessed using chi-square statistic, $\chi 2$, with low, insignificant $\chi 2$ considered a good fit [55]. The incremental fit was evaluated using Root Mean Square Errors of Approximation (RMSEA) with a value less than 0.8 indicating a relatively good fit, along with Comparative Fit Index (CFI) and Tucker-Lewis Index (TLI) with a value of 0.97 or greater considered desirable [55, 59-60]. Convergent validity among items was determined by estimating the unstandardized factor loadings and Cronbach's alpha with significant loadings and alpha of 0.70 or higher considered good reliability [55]. Construct validity of the model was evaluated by examining the completely standardized factor loadings with approximately factor loadings of 0.5 or higher and construct reliability (Cronbach's alpha) equal or greater than 0.7 considered to be a good fit [55]. Also, a parametric test of the significance of each estimated (free) coefficient was performed. Insignificant loadings with low standardized loading estimates were deleted from the model.

The completely standardized loadings were examined for offending estimates to assess problems with the model, such as loadings above 1.0. Any identified offending estimates were dropped from the model. Finally, internal consistency estimates (Cronbach's alpha) were calculated for the item representing the CIHPP outcome factor retained. Cronbach's alpha of 0.7 was considered as a minimum acceptable level of internal consistency for retaining the factor [55]. For factors with Cronbach's alpha below this minimum threshold, an attempt to improve the internal consistency was made by identifying and removing items with low item-test correlation and item-rest correlation (Nunnally \& Bernstein, 1994). If no improvement of the reliability score occurred, the factor was deleted from the model of the construct. The likelihood that the model's parameter estimates from the original sample will crossvalidate across in future samples was assessed by examining the [61] consistent version of the AIC (CAIC) with lower values of the hypothesized compared to the independent and saturated models considered to be an appropriate fit. The likelihood that the model cross-validates across similar-sized samples from the same 
population was determined by examining the Expected CrossValidation Index (ECVI) with an ECVIvalue for the hypothesized model lower compared to both the independent and saturated models considered to represent the best fit to the data. Finally, [62] Critical N (CN) was examined to determine if the study's sample size is sufficient to yield an adequate model fit for a $\chi 2$ test [59] with a value over 200 for both .05 and $.01 \mathrm{CN}$ indicative of the CIHPP outcome measurement model adequately representing the sample data [49]. The normality of the distribution of the variables in the model was assessed by Mardia's (1970; 1974) normalized estimate of multivariate kurtosis with a value of 5 or less reflexive of normal distribution. Multivariate outliers were detected by computation of the squared Mahalanobis distance (D2) for each case with D2 values standings distinctively apart from all the other D2-values indicative of an outlier

\section{Multi-group analysis}

After validating the factorial structure of NMSPIAQ, we proceeded to conduct a series of multiple groups CFA to test the invariance of CIHPP outcome factors across static factors groups. The multiple-group analysis of this study involved performing three types of CFA. First, examining the factorial invariance of CIHPP outcome factor scales (1st Order CFA Model). Second, testing the invariance of dynamic factor mean structure. Third, testing the invariance of CIHPP factors causal structure. The central concern of measurement invariance was testing measurement equivalence across groups [63]. We conducted the test at two types of models that frequently used: first-order models and second-order models (Little,1997). These tests are the recommended procedures for testing measurement invariance across a hierarchical series of models, and their common purpose is maximizing the interpretability of the results sought at each step of the hierarchy [64].

\section{Latent growth curve (LGC) modeling}

The LGC modeling within the SEM framework was relied upon to evaluate the excessive alcohol consumption risk awareness and excessive alcohol consumption of each minority young adult periodically, including indicators of progress and regression of time-invariant predictors CIHPP outcome domains in case of the presence of heterogeneity of dynamic factor variance. Unlike like the usual "scape shots" approach of taking the status of outcome domains of interest before and after an intervention such as CIHPP time-invariant and dynamic factors, the LGC model captures the actual development of the processes and outcome domains of interest following a trajectory over time to reveal the intricacies of intra-individual and inter-individual changes of the study participants. Therefore, the approach capitalizes on the richness of continuous multi-wave data to provide a somewhat superior program evaluation approach for answering questions about systematic intra-individual and inter-individual change among minority young adult CIHPP participants during 24 months [65-
67]

The next step was to assess an increase or decline in change over time for one or more CIHPP domain of interest. A representative sample of the participants was tested systematically over time, and their status in each CIHPP domain outcome was measured on several temporal-spaced occasions based on four conditions [65]. First, the outcome variable of the domain of interest must be an interval level of measurement [68-69]. Second, while the time lag between occasions can maybe evenly or unevenly spaced, both the number and spacing of these assessments must be the same for all CIHPP participants. Third, when the focus is on individual CIHPP participants, data must be obtained for each CIHPP participant on three or more occasions, and change is structured as an LGC model, with analyses conducted using the SEM procedure. Finally, the sample size must be large (i.e., a minimum 200) enough to allow for the detection of person-level effects [65] Bootsma, 2005; Bootsma \& Hoogland, 2001). Our proposed LCG model met all of these four conditions.

The basic building block of the LGC model comprised of two sub-models referred to as Level 1 model and Level 2 model [65]. Level 1 model is a within-person regression model representing an individual's change over time of the outcome variables, which in our case are the five CIHPP outcome domains mentioned earlier. Level 2 model is the between-person model that focuses on inter-individual differences in CIHPP outcome factors change over time. Level 1 (i.e., intraindividual minority young adult change) focuses on capturing the measurement model, which is the portion of the model that incorporates only linkages between the observed variables of the measurement instrument and their underlying observed or latent construct or factor (i.e., likelihood of excessive alcohol consumption). As in any measurement model, the primary interest is the strength of the factor loading or regression paths linking the observed variable to the unobserved variable. The only parts of the model that are relevant in the modeling of intraindividual change are the regression paths linking the observed variables to the unobserved factor (both intercept and slope), the factor variances and covariances, and the related measurement errors associated with these observed variables. This part of the modeling is an ordinary factor analysis model with the following two distinctive features. First, all the loadings are fixed (i.e., there are no unknown factor loadings). Second, the pattern of fixed loadings plus the mean structure allows us to interpret the factors as intercept and slope factors. As in all factor models, the present case argues that each minority young adult's likelihood of excessive alcohol consumption at each temporal time point (i.e., Time $1=0$; Time $2=1$; Time $3=2$ ), are a function of three distinct components:

- $\quad$ A factor loading matrix of constants (1:1:1) and known time values $(0: 1: 2)$ that remain invariant across all individual BOP innates, multiplied by.

- A latent growth curve vector containing particular 
minority young adult-specific and unknown factors called particular CIHPP participant growth parameter (Intercept, Slope), plus.

- $\quad$ A vector of individual minority young adult-specific and unknown errors of measurement [49]. Whereas a latent growth curve vector represents the within-person actual change in the likelihood of excessive alcohol consumption over time, the error vector represents the within-person likelihood of excessive alcohol consumption "noise" that serves to erode these actual change values [65].

Level 2 argues that, over and above the hypothesized linear change in CIHPP outcome domains over time, trajectories will necessarily vary across CIHPP participants due to differences in intercepts and slopes. Within the framework of SEM, this portion of the model reflects the "structural model" component, which in general portrays relationships among unobserved factors and postulated relations among their associated residuals. However, within the more specific LGC model, this structure is limited to the means of the Intercept and Slope factors and their related variances, which represent deviations from the means. The means carry information on individual differences in intercept and slope values. The specification of these parameters, then, makes possible the estimation of interindividual differences in change. AMOS 26.0 Graphics were used to test the latent Growth Curve Model 1 and Model 2. AMOS was also used to test the LGC. Models with static factors as a time-invariant predictor of change. This test aimed to determine if the static variable can explain statistically significant heterogeneity in the individual growth trajectories (i.e., intercept and slope) of CIHPP outcome domains as time-invariant predictors of change. Specifically, this later test aimed at answering two questions. First, "Do the CIHPP outcome domains differ for the subsets of a static factor at time 1?" second, "Do the CIHPP outcome domains change over time differ over time for a subset of a static?" The answer to these questions used the predictor "static factor," or variable incorporated into the Level 2 (or structural) path of the model. This predictor model represented an extension of our final best-fitting multiple domain model (Model 3).

The following four new structural model components were included in the measurement models. First, the regression paths that flow from the static factors to the intercept and slope factors associated with CIHPP outcome domains are of primary interest in this predictor model as they hold the key in answering the question of whether the trajectory of CIHPP outcome domains differs for the subset groups of the static factor. Second, there is now a latent residual associated with each of the intercept and slope factors. This addition is a requirement as these factors are now dependent variables in the model due to the regression paths generated from the predictor variables of the static factors. Given that dependent variables cannot be estimated in SEM, the latent factor residuals served as proxies for the intercept and slope factors in capturing the variances. These residuals represented variation in the intercepts and slopes after all variability in their prediction by the static factors has been explained [67]. Third, the covariances link the appropriate residuals rather than the factors themselves. Finally, the means of the residuals were fixed to 0.0 .

The first step in building the LGC model involved determining the direction and extent of change in outcomes of each CIHPP participant's scores over the specified time of participation in the CIHPP programming. Following [49] protocol for determining and testing the LGC model, the shape of the growth trajectory was known in advance and the LGC assumption of modeling linearity, which states that the specified model should include the following two growth parameters: (a) an intercept representing an individual CIHPP participant's domain outcome score on the outcome variable at time 1, and (b) slope parameters representing an individual CIHPP participant's rate of change throughout the period. In our study, the intercept described a CIHPP participant's CIHPP excessive alcohol consumption awareness and excessive alcohol consumption scores at the beginning of the intervention; and the slope represented the rate of change of the two constructs of interest scores over the 24-month transition. The hypothesized link between the individual growth parameter (i.e., the intercept and the slope) of levels one and level two models were considered analysis of change in the CIHPP process and outcome domains.

There are two main advantages in testing individual latent growth model LGC) change within the framework of structural equation modeling over other longitudinal approaches. First, the LGC modeling within the SEM framework evaluation approach is the analysis of mean and covariance structures and, which allows for a distinction of group effects observed in means from individual effects observed in covariance. Second, it allows for a distinction between observed and unobserved (or latent) variables in the specification of models. This capability allows for both modeling and estimation of measurement error [49]. It stands to reason that our LGC analytic approach explains the heterogeneity of interindividual differences based on one or more predictors variable, and covariates or moderators. The analysis uses AMOS version 26.0 plugins to perform these analyses.

\section{Results}

The results of this study consist of estimates of mean, covariance, and variance of the latent growth curve model of two CIHPP effectiveness in raising excessive alcohol consumption risk awareness and reducing excessive alcohol consumption. The results of each of these CIHPP outcome domains are presenting below.

\section{Effectiveness in Reducing Excessive alcohol consumption risk awareness latent growth curve model results}

Mean estimate: Results of the analysis indicate that the mean estimate of excessive alcohol consumption for the intercept and the slope are statistically significant. The results reveal that the average 
score for excessive alcohol consumption risk awareness increased (2.863) significantly over the 24-months periods, as indicated by the value of $0.374 ; p=001$. (Table $1-1$ ).

Covariance estimate: Table 1-2 shows the results of the covariance estimate between the intercept and slope factors for excessive alcohol consumption risk awareness. The results indicate that the covariance between the intercept and slope factor for excessive alcohol consumption risk awareness was statistically significant $(p=.001)$. The positive estimate of .774 suggests that minority young adults exhibited a rate of increase in their excessive alcohol consumption awareness over the 24 months. This finding indicates that the CIHPP was effective in raising the excessive alcohol consumption risk perception of young adults under study.

Variance estimate: The variance estimate related to the intercept and slope for excessive alcohol consumption risk awareness is statistically significant $(\mathrm{p}=.001)$.This finding reveals substantial inter-individual differences in the original score of excessive alcohol risk perception between the young adults at the beginning of the implementation of the CIHPP and its change over time, as the young adult progressed from the beginning of the CIHPP intervention through the 24 months. Such evidence provides powerful support for further investigation of variability related to the growth trajectory. Specifically, incorporating time-invariant change into the model explains the participants" excessive variability of alcohol consumption risk awareness. This incorporation involves testing the latent growth curve model with the demographic or static variable as a time-invariant predictor of change [49]. This study incorporated gender in the LGC model as a predictor of growth. Table 1-3 displays the result.

\section{Excessive alcohol consumption risk latent growth curve model results}

Mean estimate: The results indicate that the mean estimate of excessive alcohol consumption risk for the intercept and the slope are statistically significant. Specifically, the findings reveal that the average score for excessive alcohol consumption (-5.016)

decreased significantly over the three 6-months periods as indicated by the value of $20 / 662 ; p=001$. Hence, we can conclude that CIHPP was effective in reducing excessive alcohol consumption among minority young adults. Table 2-1.

Covariance estimate: The covariance between the intercept and slope factor for excessive alcohol consumption risk was not statistically significant $(p=.189)$. The positive sign suggests that young adults exhibited a high rate of increase in their alcohol consumption over the 24 months. This finding indicates that the Comprehensive, integrated HIV prevention program was not effective in decreasing the excessive alcohol consumption of young adults under study. Table 2-2.

Variance estimate: The variance estimate related to the intercept and slope for excessive alcohol consumption risk is statistically significant $(\mathrm{p}=.001)$. This finding reveals substantial inter-individual differences in the original score of excessive alcohol risk perception between the young adults at the beginning of the implementation of the CIHPP and its change over time, as the young adult progressed from the beginning of the CIHPP intervention through the 24 months. Such evidence provides powerful support for further investigation of variability related to the growth trajectory. Specifically, incorporating time-invariant change into the model can explain the participant's variability of alcohol consumption risk awareness. This incorporation involves testing the latent growth curve model with the demographic or static variable as a time-invariant predictor of change [49]. This study incorporated gender in the LGC model as a predictor of growth. Table 2-3 present the results of the variance estimate.

Regression Weight with Gender as Predictor: Gender was found not to be a statistically significant predictor of excessive alcohol consumption risk predictor of both initial status (-.001) at $\mathrm{p}=.981$ and rate of change $(.018)$ at $\mathrm{p}=.811$. This finding suggests that there was no meaningful difference in excessive alcohol consumption risk between minority young adult males and females both at the beginning of CIHPP and the rate of change during the 24 months intervention period. Table 2-4.

Table 1-1: Intercept and Slope Mean estimate of excessive alcohol consumption risk awareness.

\begin{tabular}{|c|c|c|c|c|c|}
\hline \multicolumn{2}{|c|}{} & Standard Error & t-value & Significance & Label \\
\hline Intercept & 2.702 & 0.044 & 61.788 & 0.001 & I Mean \\
\hline Slope & -0.192 & 0.072 & -2.648 & 0.008 & S Mean \\
\hline
\end{tabular}

Note: $\mathrm{N}=498$.

Table 1-2: Intercept and Slope Covariance Estimate for Excessive Alcohol Consumption Risk Awareness.

\begin{tabular}{|c|c|c|c|c|}
\hline & Estimate & Standard Error & t-value & Significance \\
\hline Intercept<-->Slope & 0.415 & 0.137 & 3.016 & 0.003 \\
\hline
\end{tabular}

Note: $\mathrm{N}=498$.

Table 1-3: Intercept and Slope Variance Estimate of excessive alcohol risk consumption awareness.

\begin{tabular}{|c|c|c|c|c|}
\hline & Estimate & Standard Error & t-value & Significance \\
\hline Intercept & -0.417 & 0.106 & -14.397 & 0.001 \\
\hline Slope & -628.364 & 0.266 & -2.562 & I Variance \\
\hline
\end{tabular}

Note: $\mathrm{N}=498$ 
Table 2-1: Intercept and Slope Mean Estimate for Excessive Alcohol Consumption.

\begin{tabular}{|c|c|c|c|c|c|}
\hline & Estimate & Standard Error & t-value & Significance & Label \\
\hline Intercept & -5.016 & 0.385 & -13.021 & 0.001 & I Mean \\
\hline Slope & 20.622 & 1.528 & 13.493 & 0.001 & S Slope \\
\hline
\end{tabular}

Table 2-2: Intercept and Slope Covariance Estimate for Excessive Alcohol Consumption Risk.

\begin{tabular}{|c|c|c|c|c|c|}
\hline & Estimate & Standard Error & t-value & Significance & Label \\
\hline Intercept $<--$ & -27.48 & 20.911 & -1.314 & 0.189 & Covariance \\
\hline$>$ Slope & & & & & \\
\end{tabular}

Table 2-3: Intercept and slope Variance Estimate of excessive alcohol consumption risk.

\begin{tabular}{|c|c|c|c|c|c|}
\hline & Estimate & Standard Error & t-value & Significance & Label \\
\hline Intercept & -124.333 & 8.636 & -14.397 & 0.001 & Intercept Value \\
\hline Slope & 628.364 & 17.049 & 8.263 & 0.001 & Slope Value \\
\hline
\end{tabular}

Note: $\mathrm{N}=498$.

Table 2-4: Intercept and Slope with Gender Predictor Regression Estimate of excessive alcohol consumption.

\begin{tabular}{|c|c|c|c|c|c|}
\hline & Estimate & Standard Error & t-value & Significance & Label \\
\hline Intercept & -0.001 & 0.029 & -0.024 & 0.981 & Par_5 \\
\hline Slope & 0.018 & 0.018 & 0.239 & 0.811 & Par_6 \\
\hline
\end{tabular}

Note: $N=498$

\section{Conclusions}

The Comprehensive, integrated HIV prevention program effectively raised the excessive alcohol consumption risk awareness of minority young adults under study. There is a significant interindividual difference in the original score of excessive alcohol risk perception between the young adults at the beginning of the implementation of the CIHPP and its change over time, as the young adult progressed from the beginning of the CIHPP intervention through the 24 months. Female young adult's excessive alcohol consumption risk awareness was more significant than male minority young adults. In summation, The CIHPP effectively increased the awareness of excessive alcohol consumption risk of minority young adults. The alcohol awareness of young female adults was higher than the male minority young adult during the 24 months implementation of the CIHPP. Hence, hypothesis 1.confirmed.

Regarding excessive alcohol consumption risk, the CIHPP effectively decreased excessive alcohol consumption among minority young adults. Hence, hypothesis 2 is confirmed. There was inter-individual differences or heterogeneity in alcohol consumption among the minority young adults between minority young adult at the beginning of CIHPP intervention and through the 24 months. However, there was no meaningful difference in excessive alcohol consumption between minority young adult males and females, both at the beginning of CIHPP and the rate of change during the 24 months intervention period. In other words, the interindividual difference was not attributable to gender. Collectively, the result of this study is consistent with previous studies [38,48] March \&Susser, 2006; [39,70].

\section{Study Limitations}

The study used one static variable, gender, as a predictor of excessive alcohol consumption awareness and excessive alcohol consumption risk. To more precisely evaluate interindividual change, we recommend that future studies use two or more static valuables. Also, the sample for this study was drawn from one jurisdiction, only making external validity questionable. Therefore, as a contribution to theory building, future studies two conduct the research in similar in two or more jurisdictions with similar populations. Finally, this study used a sample size of 498 minority young adults. Although this sample meets the recommended minimum threshold of a sample size of 200 for structural equation modeling [49], the sensitivity of statistical significance testing to sample size [71], we recommend that future studies should use effect size instead [72-92].

\section{Acknowledgements}

US Department of Human Services, Substance Abuse, and Mental Health Service Administration provided funding for this project (Grant Number: SP021355-01). We also express our gratitude to the staff of the Office of Sponsored Research and Programs at Fayetteville State for overseeing the successful implementation of this grant project. Finally, we acknowledge the minority young adults who participated in the survey.

\section{Conflict of Interest}

No conflict of interest.

\section{References}

1. Substance Abuse and Mental Health Services Administration (SAMHSA, 2009). Results from the 2008 National Survey on Drug Use and Health: 
National Findings (NSDUH Series H-36, HHS Publication No. SMA 094434). Rockville, USA.

2. Center for Disease Control and Prevention [CDC] (2013) Youth risk behavior surveillance in system-United States. Morbidity and Mortality Weekly Report 63(4): 1-168.

3. RachdaouI N, Sakar DK (2018) Pathophysiology of the effect of alcohol abuse on the endocrine system. Alcohol Research Current Reviews 38(2): 255-276.

4. Stephens MAC, Ward G (2018) Stress and the HPA axis: Role of Glucocorticoids in alcohol dependence. Alcohol Research: Current Reviews 34(4): 460-484.

5. Sakar DK, Gibbs DM (1984) Cyclic variation of oxytocin in the blood of pituitary portal vessels of rats. Neuroendocrinology 39(5): 481-483.

6. Plosky PM (1991) Pathway to the secretion of adrenocorticotropin: A view from the portal. Journal of Neuroendocrinology 3(1): 1-9.

7. Jenkins JS, Connolly J (1968) Androcortical response to alcohol to ethanol in man. British Medical Journal 2(5608): 804-805.

8. Uhart M, Oswald M, McCaul ME (2006) Hormonal response to psychological stress and family history of alcoholism, Neuropsychopharmacology 31(10): 2255-2263.

9. Mongkuo MYB, Mongkuo MY, Lucas N, Ayivi F, DeLone G (2020) The Impact of Health Risk Behaviors on Mental Health of Minority Young Adults Living in High HIV Prevalence Urban Community. International Journal of Psychology and Behavioral Science 14(4): 00128-00142.

10. Zimmermann U, Spring K, Knuz Ebrecht SR (2004) Effect of ethanol on hypothalamic-pituitary-adrenal system response to psychosocial stress in sons of alcohol-dependent fathers. Neuropsychopharmacology 29(6): $1156-1165$

11. Aoun EG, Lee MR, Haas Koffler CL (2015) Relationship between the thyroid axis and alcohol craving. Alcohol and Alcoholism 50(1): 24: 24 29.

12. Van Cauter E, Latta F, Ndeltcheva (2004) The reciprocal interaction between the GH axis and sleep. Growth Hormone and IGF Research 14 (Suppl A): S10-S17.

13. De Marinis L, Mancini A, Fiumara C (1993) Growth hormone response to growth-hormone-releasing hormone in early abstinence alcoholic patients. Psychoneuroendocrinology 18(7): 475-183.

14. Valimaki M, Pelknen R, Harkonen M, Ylikahri R (1984) Hormonal changes in noncirrhotic male alcoholics during ethanol withdrawal Alcohol and Alcoholism 19(3): 235-242.

15. Moller N, Jorgensen JO (2009) Effects of growth hormone on glucose, lipids, and protein metabolism in human subjects. Endocrine Reviews 30(2): 152-177.

16. Ross E, Young LJ (2009) Oxytocin and the neural mechanism regulating social cognition and affiliative behavior. Frontier of Neuroendocrinology 30(4): 534-547.

17. Insel TBR (2010) The challenge in translation in social neuroscience: $A$ review of oxytocin, vasopressin, and affiliative behavior. Neuron 65(6): 768-779.

18. Iovino M, Guastamacchia E, Giaguli VA (2012) Vasopressin secretion control: Central neural pathways, neurotransmitters, and effects of drugs. Current Pharmaceutical Design

19. McGregor IS, Bowen (2012) Breaking the loop: Oxytocin as a potential treatment for drug addiction. Hormones and Behavior 61(3): 331-339.

20. Schepisty S, Hakes Jahn K (2011) Non-medical prescription use increases the risk for the onset and recurrence of psychopathology: results from the National Epidemiological Survey on Alcohol and Related Conditions. Addiction 106(12): 2146-2155.

21. De Wied D, Diamant M, Fodor M (1993) Growth hormone response to growth hormone-releasing hormone and related peptides. Frontiers in Neuroendocrinology 14(4): 251-302.
22. Stoop R (2014) Neuromodulation by oxytocin and vasopressin in the central nervous system as a basis for their rapid behavioral effects. Current Opinion in Neurobiology 29: 187-193.

23. Richardson HN, Lee SY, O Dell, LE (2008) Alcohol self-administration acutely stimulates the hypothalamic-pituitary-adrenal axis., but alcohol dependence leads to dependence a dampened neuroendocrine state. European Journal of Neuroscience 28(8): 1641-1653.

24. Varlinskaya E, Spear LP (2004) Differences in social consequences of ethanol emerge during the course of the adolescent in rats: Social facilitation, social inhibition, andanxiolysis. Developmental Psychology 48(2): 148-161

25. Thayer JF, Hall M, Soller JJ III, Fischer JE (2006) Alcohol use, urinary cortisol, and heart rate variability in apparently healthy men: Evidence for impaired inhibitory control of the HPA axis in heavy drinkers. Internal Journal of Psychophysiology 59(3): 244-250.

26. Ross Ak, Shaw S, Predasdergast MA, and Little HJ (2010) The importance of glucocorticoids in alcohol dependence and neurotoxicity.2010. Alcoholism: Clinical and Experimental Research 34(12): 2010-2018.

27. Wand GS, Oswald LM, McCaul EM (2007) Association of amphetamineinduced striate dopamine release and cortisol responses to psychological stress. Neuropsychopharmacology 32(1): 2310-2320.

28. Koob GLA (2010) A role for brain stress system in addiction. Neuron 59(1): 11-34.

29. Hermann D, Hainz A, Mann K (1981) Dysregulation of the hypothalamicpituitary-thyroid axis in alcoholism. Addiction 97(11): 1369-1381.

30. Verbalis JG (1993) Osmotic inhibition of neurohypophysial secretion. Annals of the New York Academy of Sciences. Pp. 146-160.

31. Buijs RM, De Vries GJ, Van Leeuwen FW, Swaab DF (1983) Vasopressin and oxytocin: Distribution and putative functions of the brain. Progress in Brain Research 60: 115-122.

32. Rasmussen DD, Bolt BM, Bryant CA (2000) Chronic daily ethanol and withdrawal: 1. Long Term Changes in the hypothalamic-pituitaryadrenal axis Alcoholism. Clinical and Experimental Research 24(12): 1836-1849.

33. Rivier C, Lee S (1996) Acute alcohol administration stimulates the activity of the Hypothalamic-pituitary axis that expresses corticotropinreleasing factor and vasopressin. Brain Research 726(1-2): 1-10.

34. King A, Munisamy G, de Witt H, Lin S (2006) The attenuated cortisol response to alcohol in heavy social drinkers. International Journal of Psychophysiology 59(3): 203-209.

35. Prestage G, Jin F, Bavinton B, Hurley M (2018) Sex workers and their clients among Australian gay and bisexual men. AIDS Behavior. 18(7): 1293-1301.

36. Briere FN, Rohde P, Seeley JR, Kelin D, Lexinsohn P. et al, (2004) Comorbidity between major depression and alcohol use disorder from adolescent's adulthood. Comprehensive Psychiatry 55(3): 526-533.

37. Wu CYYS, Wu JF, Lee SY, Huang L, Yu HC Ko, et al. (2008) The association between DRD2/ANKK1, 5_HTTLPR gene, and specific personality trait on antisocial alcoholism among Han Chinese in Taiwan, American Journal of Medical Genet B Neuropsychiatry Genet 147B (4): 447-453.

38. Bronfenbrenner U (1994) Ecological models of human development. International Encyclopedia of Human Development. $2^{\text {nd }}$ Edition. Oxford, UK: Elsevier pp. 1643-1647.

39. Sudhinaraset M, Wigglesworth C, Takeuchi D (2018) Social and cultural contexts of alcohol use: Influences of a Social-Ecological Framework. Alcohol Research Current Reviews 38(1): 35-45.

40. Di Clemente RJ, Salazar LF, Crosby RA (2007) A review of STD/HIV preventive interventions for adolescents: sustaining effects using an Ecological approach. Journal of Pediatric Psychology 32(8): 888-906.

41. Jessor R (1991) Risk behavior in adolescence: a psychosocial framework for understanding and action. Journal of Adolescence Health 12(8): 597605 . 
42. Catalano RF, Hawkins JD, Berglund ML, Pollard JA, Arthur MW, et al. (2002) Prevention science and positive youth development: competitive or cooperative frameworks? Journal of Adolescent Health 31(6): 230239.

43. Ralph J DiClemente, Laura F Salazar, Richard A Crosby (2007) A review of STD/HIV preventive interventions for adolescents: sustaining effects using an Ecological approach. J Psychol 32(8): 888-906.

44. Fichtenberg CM, Muth SQ, Brown B, Padian NS, Glass TA, et al. (2009) Sexual network position and risk of sexually transmitted infection. Sex Transmision\& Infection 85(7): 493-498.

45. Gruenewald PJ, Millar AB, Treno AJ (1993) Alcohol availability and the ecology of drinking behavior. Alcohol Health \& Research World 17(1): $39-45$.

46. Gruenewald PJ (2007) The spatial ecology of alcohol problems: Niche theory and assortative drinking. Addiction 102(6): 870-878.

47. Scribner RA, Sandy A Johnson, Deborah A Cohen, William Robinson, Thomas A Farley, et al. (2008) Geospatial methods for identification of core groups for HIV/AIDS. Subst Use Misuse 43(2): 203-221.

48. Treno AJ, Grube JW, Martin SE (2003) Alcohol availability as a predictor of youth drinking and driving: A hierarchical analysis of survey and archival data. Alcoholism: Clinical and Experimental Research 27(5): 835-840.

49. Glass TA, M McAtee (2006) Behavioral science at the crossroads in public health: Extending horizons, envisioning the future. Social Science \& Medicine 62(7): 1650-1671.

50. Byrne BM (2016) Structural Equation Modeling with AMOS: Basic concept, applications, and programming $\left(2^{\text {nd }}\right.$ Edn). New York, NY: Routledge, Taylor \& Francis Group

51. Cohen L, Manion L, Morrison K (2018) Research Methods in Education. Routledge Taylor \& Francis Group, London: United Kingdom.

52. Yegidis BL, Weinbach RW, Myers LL (2018) Research Methods for Social Workers. Publishers, NY. New York

53. Isaac L, Michael WB (2014) Handbook in Research and Evaluation. EdIT Publisher, San Diego: CA, USA.

54. McNabb DE (2018) Research Methods in Public Administration and Non-Profit Management, Armonk: New York

55. Byrne BM (2008) Testing for time-invariant and time-varying predictor of self-perceived ability in math, language, and science: A look at gender factor. In HMG Watts \& IS Eccles (Eds.) Gender and Occupational outcomes: Longitudinal assessment of individual, social, and cultural influences Washington, DC: American Psychological Association. pp. 145-169.

56. Hair JF, Black WC, Babin BJ, Anderson RE, Tatham RL (2010) Multivariate Data Analysis. Pearson/Prentice-Hall, Upper Saddle River: Pearson/ Prentice Hall.

57. Hans Vaughn DL (2017) Applied Multivariate Statistical Concepts. New York, NY: Routledge: Taylor Francis Group, USA.

58. Thurstone LL (1947) Multiple-Factor Analysis. Chicago: University of Chicago Press, Chicago, USA.

59. Bunch NJ (2010) Introduction to Structural Equation Modeling Using SPSS and AMOS Los Thousand Oaks, CA.

60. Hu L, Bentler PM (1995) Evaluating model fit. In R.H. Hoyle (Ed.). Structural equation modeling: concept, issues, and applications pp. 7999.

61. Brown TA (2014) Confirmatory Factor Analysis for Applied Research. New York, NY: Guilford Press, USA.

62. Bozdogan H (1987) Model selection and Akaike's information criteria (AIC): The general theory and its analytic extensions." Psychometrika. 52: $345-370$
63. Hoelter JW (1983) The analysis of covariance structure with incomplete data: A developmental perspective. In TD. Little, J.A. Bovaird, \& NA. Card (Eds.) Modeling contextual effects in longitudinal studies. Mahwah, NJ: Erlbaum, pp. 13-32.

64. Byrne B, Shavelson R, Muthén B (1989) Testing for the equivalence of factor covariance and mean structures: The issue of partial measurement invariance. Psychol Bull 105: 456-466.

65. Meredith W (1993) Measurement invariance, factor analysis, and factorial invariance. Psychometrika 58: 525-543.

66. Willett JB, Sayer AG (1994) Using covariance structure analysis to detect correlate and predictor of individual change over time. Psychological Bulletin 116: 363-381.

67. Bryke AS, Raudenbush SW (1987) Applications of hierarchical linear models to assessing change. Psychological Bulletin 101: 147-158.

68. Willett JB (1988) Questions and answers in the measurement of change. In E.Z. Rothkopf. Review of Research in Education. Washington DC.: American Educational Association 15: 345-422.

69. Curran PJ, Edwards MC, Wirth RJ, Hussong AM, Chassin L, et al. (2007) The incorporation of categorical measurement models in the analysis of individual growth. In TD. Little \& NA. Card (Eds.), Modeling contextual effects in longitudinal studies Mahwah, NJ: Erlbaum. pp. 89-120.

70. Duncan TE, Duncan SC, Stryker LA (2006) An introduction to latent variable growth curve modeling Concepts, issues, and applications $\left(2^{\text {nd }}\right.$ Edn). Mahwah, NJ: Elbaum.

71. Dahlberg LL, Krug EG (2002) Violence: A global public health problem. In: Krug E, Dahlberg LL, Mercy JA, Lozano R, editors. World Report on Violence and Health. Geneva, Switzerland: World Health Organization. pp. 1-56.

72. Cumming G, Calin Jageman (2017) Introduction to the New Statistics: Estimation, Open Science, \& beyond. New York: Routledge Taylor 7 Francis Group.

73. Brenner ND, Collins JL (1998) Co-occurrence health risk behaviors among adolescents in the United States. Journal of Adolescence Health. 22 (3): 209-213.

74. Byrne BM, Crombie G (2003) Modeling and testing change over time: An Introduction to the growth curve model. Understanding Statistics: Statistical Issues in Psychology, Education, and the Social Sciences 2: 177-203.

75. Ferguson C, Meehan D (2011) With a friend like these: Peer delinquency influences across age cohorts on smoking, alcohol, and illegal substance use. European Psychiatry 26: 6-12.

76. Daban C, Vieta E, Mackin P, Young AH (2005) The hypothalamicpituitary-adrenal axis and bipolar disorder. The Psychiatric clinics of North America 28 (2): 469-80.

77. Dahlberg LL, Krug EG (2002) Violence: A global public health problem. In: Krug E, Dahlberg LL, Mercy JA, Lozano R, editors. World report on violence and health. Geneva, Switzerland: World Health Organization. pp. 1-56.

78. Elkins IJ, McGue M, Iacono WG (2007) Prospective effect of attentiondeficit/hyperactivity disorder, conduct disorder, and sex on adolescent substance use and abuse. Archives of General Psychiatry 64: 1145-1152.

79. Glossary of Terms for Nebraska Behavioral Health System (2006) Mental Health board Training Self-Study.

80. Goodman I, Peterson Badali M, Henderson J (2011) Understanding substance treatment: The role of social pressure during the transition to adulthood. Addictive Behaviors 36(6): 660-668.

81. Jessor R (1991) Risk behavior in adolescence: a psychosocial framework for understanding and action. Journal of Adolescence Health 12 (8): 597605. 
82. Rasmussen DD, Bolt BM, Bryant CA (2000) Chronic daily ethanol and withdrawal: 1. Long Term Changes in the hypothalamic-pituitaryadrenal axis Alcoholism: Clinical and Experimental Research 24(12): 1836-1849.

83. Ross HE, Young LJ (2009) Oxytocin and the neural mechanisms regulating cognitive and affiliative behavior. Frontiers in Neuroendocrinology 30(4): 534-547.

84. Substance Abuse and Mental Health Services Administration (SAMHSA, 2009). Results from the 2008 National Survey on Drug Use and Health: National Findings (NSDUH Series H-36, HHS Publication No. SMA 094434). Rockville, USA.

85. Stephens MAC, Ward G (2018) Stress and the HPA axis: Role of Glucocorticoids in alcohol dependence. Alcohol Research: Current Reviews pp. 460-484.

86. Scribner R, Theheal KP, Simonsen N, Robinson W (2010) HIV risk and the alcohol environment: Advancing ecological epidemiology for HIV/ AIDS. Alcohol Research \& Health 33(3): 179-183.

87. Treno AJ, Grube JW, Martin SE (2003) Alcohol availability as a predictor of youth drinking and driving: A hierarchical analysis of survey and archival data. Alcoholism: Clinical and Experimental Research 27(5): 835-840.

88. Upmark M, Moller J, Romelsjo A (1999) A longitudinal population-based study of self-reported alcohol habits, high level of sickness absence, and disability pension. Journal of Epistemology. Community Health 53(4): 223-229.

89. Wand GS (2008) The influence of stress on the transition from drug use to addiction. Alcohol Research \& Health 31(2): 119-136.

90. Wang J, Wang X (2012) Structural Equation Modeling Application Using Mplus. Chichester: John Wiley \& Sons Ltd.

91. Widaman KF (2018) Common Factor Analysis Versus Principal Components Analysis: Differential Bias in Representing Model Parameters? Available online.

92. Willett JB, Sayer AG (1996) Cross-Domain analysis of change over time: Combining growth modeling and covariate structure analysis, In Modeling: Issues and Techniques. Pp. 125-157. 